\title{
Stromal Changes in Benign and Malignant Salivary Gland Tumors with Picrosirius Red-A Polarizing Microscopic Study
}

\author{
Sajda Khan Gajdhar', Shaiq Gajdhar' ${ }^{2}$, Rahul B Patil ${ }^{3}$ and Smita Chaware \\ 'Department of Oral Basic and Clinical Sciences, Ibn Sina National College for Medical Studies, \\ Jeddah 22421, Kingdom of Saudi Arabia; saajda@rediffmail.com \\ ${ }^{2}$ Department of Oral and Maxillofacial Rehabilitation, Ibn Sina National College for Medical Studies, \\ Jeddah 22421, Kingdom of Saudi Arabia; drshaiquegajdhar@gmail.com \\ ${ }^{3}$ Department of Oral pathology and Microbiology, Rural dental college, Loni - 414001, \\ Maharashtra, India; drrahulpatil79@gmail.com \\ ${ }^{4}$ Department of Oral Pathology and Microbiology, Maharashtra Institute of Dental Sciences \& Research (MIDSR) \\ Dental College, Latur-413531, Maharashtra, India; smitachaware123@gmail.com
}

\begin{abstract}
Objectives: To explore the polarizing color profile in connective tissue stroma of Picro-Sirius Red (PSR) stained sections of benign and malignant salivary gland tumors (SGT) and to assess the possible utility of this method as a diagnostic tool. Materials and Methods: A total number of 60 Paraffin blocks of benign (30 cases) and malignant ( 30 cases) salivary gland tumors were selected from the archives of our institute and regional cancer hospital. 30 cases of Mucous Extravasation Phenomenon (MEP) were selected as control. Salivary gland tumors and control were stained with PSR stain and examined under polarizing microscope. We counted 50 thin and 50 thick collagen fibers in each section of benign and malignant SGT and were categorized as Greenish Yellow (GY) or Yellowish Orange (YO). Statistical analysis was performed by using oneway ANOVA and Paired t-test. Results: We found similar presentation of polarizing color arrangement for thin collagen fibers $(80-85 \%$ GY and $15-20 \%$ YO) in both benign and malignant SGT and MEP. In malignant SGT, thick collagen fibers exhibited different polarizing color (80\% GY) than benign SGT and MEP (12-14\% GY). Polarizing color profile of collagen in malignant SGT displayed a different presentation as compared to benign SGT and control (MEP) and differences were statistically significant $(p<0.05)$. Conclusion: This study concluded about the difference of stromal collagen fibers in benign and malignant SGT. Malignant SGT suggesting loosely arranged collagen fibers could be involved in further growth and invasion of disease along with other cellular and molecular events.
\end{abstract}

Keywords: Collagen, Mucous Extravasation Phenomenon, Picro-Sirius Red (PSR) Stain, Polarized Microscopy, Salivary Gland Tumor

\section{Introduction}

Salivary gland tumors characterize the most complicated and diverse cluster of tumors seen in the head and neck. Salivary gland tumors often presents a wide spectrum of tumor histomorphology, the neoplastic lesions are too numerous, histologically most heterogeneous and present a diverse clinical behavior. The histomorphogenesis of salivary gland tumors are complex and poorly understood $^{1}$. The most common benign SGT are Pleomorphic Adenoma (PA) and Warthin's Tumors (WT), comprising $75-90 \%$ of all salivary gland tumors. Most common malignant SGT are chiefly Adenoid Cystic Carcinoma (ADCC) (30\%) and Muco-Epidermoid Carcinoma (MEC) $(70 \%)^{2}$. Numerous theories are suggested and described in literature about histogenesis of SGT. According to bi-cellular 
theory, salivary gland neoplasms are composed of: (a) Ductal luminal and/or acinar cells plus myoepithelial cells (b) Composed primarily of ductal luminal cells or acinar cells (c) Composed almost entirely of myoepithelial cells which includes components of basal lamina, collagen, elastic fibers \& glycosaminoglycans ${ }^{3}$. However, histogenesis of Warthin's tumor remains controversial. Currently most accepted concept regarding WT is that the tumor arises from the remnant of salivary gland tissue entrapped in intra or peri-parotid lymph nodes during embryological development ${ }^{2}$.These facts could improve our understanding of the diverse histological growth patterns within a single tumor type and the overlap in histological features among different tumor types. Although different tumor types share similar histological patterns their biological properties can differ ${ }^{3}$. To recognize the biological nature of SGT, studies on the collagen changes have been employed by various techniques ranging from histochemistry, immunohistochemistry, electron microscopy and PCR. Various histochemical techniques employed to study collagen are Masson Trichrome, Van Geison's, and PSR.

PSR, an anionic dye that selectively reacts and stains collagen fibers, is been of specific importance in differentiating different types of collagen fibers in many oral lesions including salivary gland tumors ${ }^{4}$.

Collagen is an anisotropic material, its profile of arrangement and nature can be examined in connective tissue stroma of benign and malignant SGT by polarizing microscope. Collagen exhibits positive birefringence. Birefringence is a phenomena exhibited by anisotropic objects which can be selectively visualized by polarizing light microscopy ${ }^{5}$. The color profile and intensity of birefringence of collagen fibers depends on thickness and molecular packing of their fibers. Thin collagen fibers are usually displayed green to GY polarizing color profile and thick collagen fibers demonstrated yellow to YO profile ${ }^{6}$. This study was planned to evaluate the stromal differences between the benign and malignant SGT by using PSR and polarizing light microscopy and thereby predict their biological behavior.

\section{Materials and Methods}

\subsection{Study Cases}

This study included the sixty paraffin embedded tissue blocks of benign (30 cases) and malignant (30 cases)
SGT. 30 cases of MEP was taken as control. SGT and MEP were retrieved from archives of oral pathology and microbiology department, M.G.V's K.B.H dental College and hospital, Nasik, Maharashtra, India and regional cancer hospital. Benign salivary gland tumors, 15 Warthin's tumor (WT) and 15 Pleomorphic Adenoma (PA) cases were selected. 15 MEC and 15 ADCC cases were included as malignant SGT in present study. 30 paraffin embedded tissue blocks of mucous extravasation phenomenon were taken as control. Two oral pathologists reevaluated the samples and confirmed the diagnoses.

\subsection{Staining Procedure}

Two sections of benign, malignant SGT and MEP of 5 $\mu$ thickness were obtained from formalin fixed paraffin embedded tissues using a semi-automated microtome (Thermo scientifics, Microm HM 340E). Hematoxylin and eosinstaining was performed with one section and other section was stained with PSR (NovaUltra stain kit). Subsequent deparaffinization and hydration of section in distilled water, it was incubated in $0.1 \%(\mathrm{w} / \mathrm{v})$ Sirius red F3B (C.I.35782) in saturated picric acid solution for one hour at room temperature. After this step, the section was dipped in distilled water followed by staining with Mayer's haematoxylin, differentiation in $1 \% \mathrm{HCl}$, alkalinization with tap water, dehydration and mounting of the stained section. PSR stained sections were observed under the polarizing microscope. Variations in the polarizing color profile of collagen fibers were noted in benign, malignant SGT and MEP. An Olympus BX 51 trinocular research microscope with polarizer was used for present study. Collagen fiber thickness was calculated with LYNX BIOLUX auto software under an oil immersion 100X objective.

Fibers with thickness of $0.8 \mu \mathrm{m}$ or less were considered as thin fibers and fibers with thickness of 1.6 to 2.4 $\mu \mathrm{m}$ were assigned as thick fibers. Polarization color profiles were recorded individually for thin and thick fibers in SGT and MEP. For each slide 50 thick and 50 thin fibers were counted in five randomly selected field and the polarizing colors of the fibers were recorded as GY or YO.

\subsection{Statistical Analysis}

Two investigators independently evaluated the cases to assess the reliability of the counts. Analysis of polarizing color variations for thick and thin collagen fibers in SGT and MEP were performed by one way ANOVA. The sig- 
nificant polarizing color differences between thick and thin collagen fibers were analyzed by using paired t-test.

\section{Results}

The present study revealed that there was a significant difference in polarizing color profile of malignant and benign SGT. Polarization color of stromal fibers of benign SGT were mostly in range of YO. Polarization color profile of malignant SGT were mainly in the range of GY whereas, in control (MEP) polarization color were found to be YO. We observed a similar GY polarization color profile for thin collagen fibers for SGT and control (80-85\%), although $15-20 \%$ was YO in range. The same pattern was observed for the reactive MEP. No significant statistical differences were found among thin collagen fibers of benign SGT, malignant SGT and control $(\mathrm{p}>0.05)$. The polarization colors profile for thick collagen fibers, demonstrated an altered pattern in the malignant tumors, ADCC and MEC, as compared to the benign tumor, PA and WT. In the malignant SGT, approximately $80 \%$ of the thick fibers were displayed GY while, in benign SGT most of the thick fibers were YO (86\%) only $14 \%$ fibers were GY. A similar pattern like benign SGT is observed in control group (MEP), where majority of thick fibers showed YO color profile. Our study results revealed a statistically significant differences in polarizing color profile of thin collagen fibers between benign and malignant SGT. $(\mathrm{p}<0.05)$.

\section{Discussion}

In majority of the studies, the neoplastic epithelial cells of SGT have been investigated for factors that contribute to the tumorigenic potential and different degrees of aggressiveness $^{7-9}$. Recently, in the field of tumorigenesis, there has been increased interest regarding the stromal components and their role in amplifying or inhibiting tumor progression ${ }^{10}$. Very few studies have investigated the importance of stromal components and its role with regard to salivary gland tumors ${ }^{3}$.

Collagen fibers are major organic constitute of connective tissue stroma. It is an anisotropic structure that exhibited a diverse interference color and intensities of birefringence, when visualized by polarizing light microscopy $^{11}$. The present study revealed PSR stained collagen fibers exhibited the birefringence, elucidating that PCR is a selective stain for collagen. Picrosirius red polarization method demonstrates fibers of different thickness with different colors ranging from greenish yellow to yellowish orange. Type-I Collage fibers are composed of tightly packed thick fibers and shows strong birefringence with yellow to red polarizing color, while type-III collagen fibers are composed of loosely arranged thin fibers and displayed a week birefringence with green to greenish yellow color. This difference in polarizing color profile of thick and thin fibers are due to different pattern of physical aggregation of collagen fibers. This is accordance with several other studies ${ }^{11-15}$. Our study also demonstrated, the polarizing color profile in benign SGT (WT and PA) and reactive lesion (MEP) were different from those of malignant SGT (MEC and ADCC). We observed similar polarization color profile for thin collagen fibers in all types of examined tumors and control, exhibiting mostly GY. Conversely, the polarizing color profile of thick collagen fibers in the malignant SGT differed significantly from the benign SGT (PA and WT) and reactive lesion (MEP). So, we could conclude that malignant SGT exhibit a different type of collagen fibers as they displayed a different pattern of birefringence suggesting, these fibers were loosely arranged and probably composed of procollagen, intermediate or pathological collagen compared to thick fibers of benign SGT and MEP. This outcome is based on the results of the previous researches, where they differentiate procollagen, intermediate or pathological collagens on the basis of collagen thickness in PSR stained sections ${ }^{16}$. One study stated that the pathological collagen fibers are not closely packed they are arranged loosely in tissue section as compared to tightly packed normal collagen fibers ${ }^{16}$.

These results are accordance with the previous studies where majority of collagen fibers exhibited green to greenish yellow color of thin collagen fibers in different pathologies such as oral submucous fibrosis, odontogenic cysts, connective tissue lesions, ameloblastic fibroma and oral squamous cell carcinoma ${ }^{17-21}$. The present study could indicate that the collagen fibers in the stroma of SGT are variable and depend on whether the tumor is benign or malignant. This variability may be explained by the concept that a benign tumor uses specific mechanisms to expand, which differ from those of a malignant tumor and requires more aggressive means for invasion and metastasis ${ }^{10}$. Thus, it is anticipated that the loosely packed collagen fibers in malignant SGT, may facilitate further invasion and metastases along with other molecu- 
lar and cellular events. These results are accordance with a study where the PSR stained stromal collagen fibers were greenish yellow at the site of invasion in follicular carcinoma of thyroid ${ }^{22}$.

\section{Conclusion}

In our study, an observable difference in stromal collagen fibers of benign and malignant SGT was evinced with PSR polarization method. PSR stained collagen fibers in malignant SGT exhibited a significant difference as compared to benign SGT and MEP. We concluded that PSR polarization method could be potentially useful method for revealing the biological behavior and pathological nature of collagen in connective tissue stroma of SGT.

\section{References}

1. Shrestha P, Huang J W, Takai Y, Mori M. Primary epithelial tumors of salivary glands - Histogenesis, Histomorphological and Immunohistochemical implications -diagnosis and clinical management, Critical Reviews in Oncology/ Hematology. 1996; 23:239-60. https://doi. org/10.1016/1040-8428(96)00203-X.

2. Shafer's textbook of oral pathology. Date accessed: 2009. https://books.google.co.in/books?id=Spk0V6TrCggC\&pri $\mathrm{ntsec}=$ frontcover $\# \mathrm{v}=$ onepage \&q\&f$=$ false .

3. Allon. Stromal differences in salivary gland tumors of a common hispathologenesis but with different biological behaviour: A study with picrosirius red and polarising microscopy, Actahistochemica. 2006; 108:259-64. https:// doi.org/10.1016/j.acthis.2006.05.007. PMid: 16899283.

4. Slootweg PJ. Epithelio-mesenchymal morphology in ameloblastic fibrodontoma a light and electron microscopic study, Journal of Oral Pathology and Medicine. 1980; 9:29-40. https://doi.org/10.1111/j.1600-0714.1980. tb01385.x.

5. Montes GS, JunqueirL CU. The use of the picrosirius - polarization method for the study of biopathology of collagen, Memórias do Instituto Oswaldo Cruz. 1991; 111:1-11. https://doi.org/10.1590/S0074-02761991000700002.

6. Venigella A, Charu S. Evaluation of collagen in different grades of Oral Squamous cell Carcinoma by using the Picrosirius red stain- a histochemical study, Journal of Clinical and Diagnostic Research. 2010; 4:3444-49.

7. Giannoni C, El-Naggar AK, Ordonez NG, Tu ZN, Austin J, Luna MA. C-erbB-2/neu oncogene and Ki-67 analysis in the assessment of palatal salivary gland neoplasms, Otolaryngol Head Neck Surg. 1995; 112:391-98. https:// doi.org/10.1016/S0194-5998(95)70272-5.
8. Rosa JC, Felix A, Fonseca I, Soares J. Immuno expression of c-erbB-2 and p53 in benign and malignant salivary neoplasms with myoepithelial differentiation, Journal of Clinical Pathology. 1997; 50:661-63. https:// doi.org/10.1136/jcp.50.8.661. PMid: 9301549, PMCid: PMC500110.

9. Edwards PC, Bhuiya T, Kelsch RD. Assessment of p63 expression in the salivary gland neoplasms adenoid cystic carcinoma, polymorphous low-grade adenocarcinoma, and basal cell and canalicular adenomas, Oral Surgery, Oral Medicine, Oral Pathology and Oral Radiology. 2004; 97:613-19. https://doi.org/10.1016/j.tripleo.2003.09.010.

10. De Wever O, Mareel M. Role of tissue stroma in cancer cell invasion, Journal of Pathology. 2003; 200:429-47. https:// doi.org/10.1002/path.1398. PMid: 12845611.

11. Shetty A, Tamgadge A, Bhalerao S, Periera T, Tamgadge S, Gotmare S. Study of polarization colors in the connective tissue wall of odontogenic cysts using picrosirius red stain, Journal of Orofacial Sciences. 2015; 7:119-24. https://doi. org/10.4103/0975-8844.169771.

12. Mahajan AM, Mahajan MC, Ganvir SM, Hazare VK. The role of stroma in the expansion of odontogenic cysts and adenomatoid odontogenic tumor: A polarized microscopy study, Journal of Natural Science, Biology and Medicine. 2013; 4(2):316-20. https://doi.org/10.4103/0976-9668.116988. PMid: 24082724, PMCid: PMC3783772.

13. Sweat F, Puchtler H. Sirius red F3BA as a stain for connective tissue, Archives of Pathology and Laboratory Medicine. 1964; 78:69-72.

14. Constantine VS, Mowry RW. Selective staining of human dermal collagen II. The use of picrosirius red F3BA with polarization microscopy, Journal of Investigative Dermatology. 1968; 50:419-23. https://doi.org/10.1038/jid.1968.68, https:// doi.org/10.1038/jid.1968.67. PMid: 4172462.

15. Junqueira LC, Bignolas G, Brentani RR. Picrosirius staining plus polarization microscopy, a specific method for collagen detection in tissue sections, Histochemical Journal. 1979; 11:447-55. https://doi.org/10.1007/BF01002772. PMid: 91593.

16. Dayan D, Hiss Y, Hirshberg A, Bubis JJ, Wolman M. Are the polarization colors of picrosirius red-stained collagen determined only by the diameter of the fibers? Histochemistry. 1989; 93:27-29. https://doi.org/10.1007/ BF00266843. PMid: 2482274.

17. Trau H, Dayan D, Hirschberg A, Hiss Y, Bubis JJ, Wolman M. Connective tissue nevi collagens. Study with picrosirius red and polarizing microscopy, American Journal of Dermatopathology. 1991; 13:374-77. https://doi. org/10.1097/00000372-199108000-00008. PMid: 1718184.

18. Dayan D, Waner T, Tal H, Nyska A. Polarization microscopy of picrosirius red stained collagen from oxodipine 
induced hyperplastic gingival of beagle dogs, International Journal of Experimental Pathology. 1993; 74:225-58. PMid: 8334073, PMCid: PMC2002159.

19. Nyska A, Dayan D. Ameloblastic fibroma in a young cat, Journal of Oral Pathology and Medicine. 1995; 24:233-36. https://doi. org/10.1111/j.1600-0714.1995.tb01173.x. PMid:7616463.

20. Hirshberg A, Buchner A, Dayan D. The central odontogenic fibroma and hyperplastic dental follicle. Study with picrosirius red and polarizing microscopy, Journal of Oral Pathology and Medicine. 1996; 25:125-27. https://doi. org/10.1111/j.1600-0714.1996.tb00206.x.
21. Hirshberg A, Sherman S, Buchner A, Dayan D. Collagen fibres in the wall of odontogenic keratocystsa study with picrosirius red and polarizing microscopy, Journal of Oral Pathology and Medicine. 1999; 28:410-12. https://doi.org/10.1111/j.1600-0714.1999.tb02112.x. PMid: 10535364.

22. oren R, Yaniv E, Kristt D, Shvero J, Veltman V, Grushko I. Capsular collagen staining of follicular thyroid neoplasms by picrosirius red: Role in differential diagnosis, Acta Histochemistry. 2001; 103:151-57 https://doi.org/10.1078/0065-1281-00587. 